\title{
The reliability and validity of the weight-bearing lunge test in a Congenital Talipes Equinovarus population (CTEV)
}

\author{
Georgia Gosse ${ }^{\text {Corresp., } 1}$, Emily Ward ${ }^{1}$, Auburn McIntyre ${ }^{2}$, Helen Banwell ${ }^{1,3}$ \\ ${ }^{1}$ School of Health Sciences, University of South Australia, Adelaide, SA, Australia \\ 2 Paediatric Allied Health Department, Women's and Children's Hospital, Adelaide, SA, Australia \\ 3 International Centre for Allied Health Evidence, University of South Australia, Adelaide, SA, Australia \\ Corresponding Author: Georgia Gosse \\ Email address: gosge001@mymail.unisa.edu.au
}

Question: What is the intra and inter-rater reliability and concurrent validity of the weight-bearing lunge test within a Congenital Talipes Equinovarus population?

Design: Test retest design for reliability and validity. The measure was taken, following preconditioning of the participants, using distance from wall, angle at distal posterior tibia using a digital inclinometer and the iPhone level function, twice by each rater. The raters included a clinician, clinician in training and a parent/carer.

Outcome measures: Weight bearing lunge test as a measure of ankle dorsiflexion.

Results: Twelve children aged 5-10 years were eligible to participate and consented, along with their parents. Intra-reliability of distance measures for all raters were good to excellent (ICC clinician 0.95, ICC training clinician 0.98 and ICC parent 0.89 ). Intra-rater reliability of the iPhone for all raters was good (ICCS > 0.751) and good to excellent for the inclinometer (ICC clinician 0.87, ICC training clinician 0.90). Concurrent validity between the clinician's and parents distance measure was also high with ICC of 0.899. Inter-rater reliability was excellent for distance measure (ICC $=0.948)$, good for the inclinometer (ICC $=0.801)$ and moderate for the iPhone $(I C C=0.68)$. Standard error of measurement ranged from 0.70-2.05, whilst the minimal detectable change ranged from 1.90-5.70.

Conclusion: The use of the WBLT within this CTEV population has demonstrated good to excellent reliability and validity amongst clinicians, clinicians in training and parents/carers, supporting its use as an assessment measure of dorsiflexion range of motion. There is support for parents/carers to use the WBLT at home as a monitoring assessment measure which may assist with early detection of a relapse.

Trial registration: University of South Australia's ethics committee (ID: 201397); Women's and Children's Hospital ethics committee (AU/1/4BD7310). 
1

"The reliability and validity of

the weight-bearing lunge test in

a paediatric Congenital Talipes

Equinovarus (CTEV)
6

7

8

9

\section{population"}

\section{Authors:}

Georgia Elder Gosse ${ }^{1}$, Emily Ward ${ }^{1}$, Auburn McIntyre ${ }^{1,2}$, Helen Banwell ${ }^{1,3}$

1. School of Health Sciences, University of South Australia, Adelaide, Australia

2. Paediatric Allied Health Department, Women's and Children's Hospital, Adelaide, Australia

3. International Centre for Allied Health Evidence, University of South Australia, Adelaide, Australia

\section{Corresponding Author: Georgia Gosse}

Email address: gosge001@mymail.unisa.edu.au 
29 Abstract

30

Question: What is the intra and inter-rater reliability and concurrent validity of the weightbearing lunge test within a Congenital Talipes Equinovarus population?

33

Design: Test retest design for reliability and validity. The measure was taken, following preconditioning of the participants, using distance from wall, angle at distal posterior tibia using 36 a digital inclinometer and the iPhone level function, twice by each rater. The raters included a

Outcome measures: Weight bearing lunge test as a measure of ankle dorsiflexion. 40

Results: Twelve children aged 5-10 years were eligible to participate and consented, along with their parents. Intra-reliability of distance measures for all raters were good to excellent (ICC 43 clinician 0.95 , ICC training clinician 0.98 and ICC parent 0.89 ). Intra-rater reliability of the iPhone for all raters was good (ICCs $>0.751)$ and good to excellent for the inclinometer (ICC clinician 0.87 , ICC training clinician 0.90). Concurrent validity between the clinician's and parents distance measure was also high with ICC of 0.899. Inter-rater reliability was excellent for distance measure $(\mathrm{ICC}=0.948)$, good for the inclinometer $(\mathrm{ICC}=0.801)$ and moderate for the iPhone $(\mathrm{ICC}=0.68)$. Standard error of measurement ranged from $0.70-2.05$, whilst the minimal detectable change ranged from 1.90-5.70.

50

Conclusion: The use of the WBLT within this CTEV population has demonstrated good to excellent reliability and validity amongst clinicians, clinicians in training and parents/carers, supporting its use as an assessment measure of dorsiflexion range of motion. There is support for parents/carers to use the WBLT at home as a monitoring assessment measure which may assist with early detection of a relapse. and Children's Hospital ethics committee (AU/1/4BD7310).

59

60 
61

62

63

64

65

66

67

68

69

70

71

72

73

74

75

76

77

78

79

80

81

82

83

84

85

86

87

88

89

90

91

92

\section{Background}

Congenital Talipes Equinovarus (CTEV), frequently known as clubfoot, is a congenital, idiopathic abnormality affecting the lower limb in newborns. ${ }^{1,2}$ Global prevalence of CTEV is approximated at 1.2 per 1000 livebirths, with a male to female predilection of approximately 2.4:1. ${ }^{3}$ Within Australia, the Aboriginal and Torres Strait Islander population experiences a greater prevalence with 3.5 per 1000 livebirths compared to 1.1 per 1000 within a Caucasian population. ${ }^{1}$ This condition causes the foot to be in an 'equinovarus' foot posture with adductus and cavus deformities also present. ${ }^{4-6}$

Management of CTEV via the Ponseti method includes a serial casting process of approximately six-weeks, followed by a percutaneous elongation of tendo-achilles and finally a bracing period lasting until age four. ${ }^{5}$ Unfortunately, the relapse rate remains a significant problem within this population with rates ranging from $5 \%$ to $68 \%$, more frequently observed in those unable to comply with the bracing protocol. ${ }^{5,7}$ One study reported that at age two, the relapse rate was $30 \% .{ }^{8}$ By the time the child was four, this was then $45 \%$ and $52 \%$ by age six. ${ }^{8}$

One of the primary signs of relapse is a reduction in ankle joint range of motion (ROM). ${ }^{5}$ The weight-bearing lunge test (WBLT), is a commonly used measure of ankle ROM (Figure 1). ${ }^{9}$ This test has been determined as reliable within healthy adult and paediatric populations as well as some pathological groups including Charcot-Marie Tooth. ${ }^{9-11}$

Monitoring of children with CTEV by health professionals decreases exponentially over time, therefore raising concern that the identification of changes in ankle joint ROM may be delayed. ${ }^{5}$ Ideally, ankle joint ROM would be assessed regularly, more frequently than standard monitoring allows, to avoid delays in identifying those requiring further intervention. ${ }^{8}$ It has been reported that the use of self-management in families enhances adherence to treatment plans and provides families with greater abilities to solve problems. ${ }^{12}$ This raises the consideration that parent/carers may be useful in early identification of relapses. 
93 The WBLT can be measured in a variety of different ways, all with reported reliability and/or 94 validity. In healthy adults, this test originally was investigated for reliability using a toe to wall 95 measure and an angular measurement along the anterior tibia. ${ }^{9}$ Another study, investigating the 96 use of the Tiltmeter App, used the angle at the posterior tibia, measuring when the knee was both 97 extended and flexed. ${ }^{13}$ This study determined good to excellent reliability and validity comparing 98 a now outdated iPhone application (the Tiltometer) with a digital inclinometer in a healthy adult 99 population. This outcome was recently repeated using the new level function of the measure 100 application, available within the Apple suite (Apple Inc., Cupertino, CA, USA), also with 101 reported good to excellent reliability within a healthy adult population. ${ }^{14} \mathrm{With}$ the increase in

102

103

104

105

106

107

108

109

110

111

112

113

114

115

116

117

118

119

120

121

122 Raters

123 Three raters conducted each measurement. The clinician and clinician in training (AM and GG)

124 were consistent for each participant, the third rater, a parent/carer, was unique to each 
125 participant. The clinician (AM) had thirty years clinical experience with specific involvement in

126 paediatric orthopaedics for approximately seven years, where the WBLT is often used in

127 practice. The clinician in training (GG) was a final year undergraduate student and had been

128 trained in the procedure within the previous six months. The parent/carers were not familiar with

129 the measure but were given explanations on how to perform the test and had the opportunity to

130 observe the raters prior to each of their measures.

131

132 The clinician and clinician in training were involved in the development of the protocol. To 133 allow for testing and revision of protocol, the study was piloted twice (at six months and one 134 week) prior to commencing formal study on a child with typical development.

135

136

137

138

139

140

141

142

143 144

145

146

147

148

149

150

151

152

153

154

\section{Participants}

A sample of convenience was recruited from the Women's and Children's Hospital (Adelaide, South Australia) Physiotherapy outpatient clinic. Potential participants were identified and informed of the study by the treating clinician via a phone call or conversation when they were present for an appointment. A participant information pack was supplied where interest was indicated. Written informed consent was obtained from the parent and verbal assent gained from the child prior to commencing the measures. Participants were informed of their right to withdraw from the study via written and verbal notification.

Inclusion criteria included children aged 4-18 years born with unilateral or bilateral idiopathic CTEV, managed via the Ponseti method. The children also were required to be able to perform a WBLT without pain and have a parent/carer able to be present and conduct measures. Exclusion criteria included current pain or lower limb injury, an inability to perform the WBLT or a parent unable to measure. Reasons for being unable to measure included inability to assume a measuring posture on the floor or other physical limitations, impaired cognitive ability or previous experience in the WBLT. A sample of $n=13$ was calculated to power the study in order to obtain $80 \%$ power, or 0.8 , to detect an Intraclass Correlation Coefficient (ICC) of $\geq 0.75$ with a desired confidence interval width of $0.5(0.5-1.0) .{ }^{16}$ 
155 In the event of a child presenting with bilateral CTEV, both feet were used as separate

156 participant data when two parents/carers were present, willing and able to measure, ensuring

157 each parent/carer was a unique rater.

158

159 The protocol was approved by the University of South Australia Human Research Ethics

160 Committee (approval 201397) and the Women's and Children's Hospital Research Ethics

161 Committee (approval AU/1/4BD7310).

162

163 Procedure

164 The tools used within the study included the Geo Fennel S-Digit Mini Inclinometer (digital 165 inclinometer), (GSR Laser Tools, Perth, Australia) and the inclinometer function within the 166 iPhone Measure application. This application is free and automatically installed on the iPhone 167 smartphone (iOS 7 and above). Within this study, an iPhone 8 was used (Apple Inc., Cupertino, 168 CA, USA). Prior to beginning the study, the digital inclinometer and iPhone Measure application 169 were compared for consistency on identical, hard flat and angled surfaces across three trials.

170 During the study the digital inclinometer was calibrated in accordance to industry requirements 171 (Laser-Liner, UK), whilst the iPhone was calibrated to zero degrees by placing it on the long axis 172 on the floor.

173

174 For the participants convenience, testing was conducted in conjunction to scheduled

175 appointments. Preconditioning required participants to perform a WBLT stance for 30 seconds, 176 three times, to demonstrate understanding of the technique and reduce joint stiffness. A small 177 mark was made on the back of the child's heel to indicate one centimeter superior to the 178 posterior calcaneal tuberosity as this was the point of measurement. ${ }^{13}$ The WBLT was 179 performed using a modified version of methods described by previous studies and Figure 2 180 shows the position in which the measure was taken. ${ }^{9}$

181

182 FIGURE 1 APPROXIMATED HERE

183

184 The measures taken included;

185 Clinician/Clinician in training: 
186

187

188

189

190

191

192

193

194 Figure 2 describes the protocol of measures.

1) Distance of hallux from wall (in millimetres);

\section{Parent/carer:}

1) Distance of hallux from wall (in millimetres);

2) Angle at back of the shin with digital inclinometer (degrees);

3) Angle at back of the shin with iPhone Measure app inclinometer (degrees).

2) Angle at back of shin with iPhone measure app inclinometer (degrees).

\section{FIGURE 2 APPROXIMATED HERE}

Unilateral CTEV participants used their affected foot. Bilateral CTEV participants with only one rater available used the foot with the higher birth Pirani score or in the case of equal scores, the child's preferred foot.

\section{The order in which the measurements were taken were pseudo-randomised via computer} programming and sealed in an envelope and labelled to corresponding participant number. For the purpose of training, the parents/carers were always the third rater. The order of the clinician and clinician in training, along with the order of measures was randomised.

The distance measure was marked on a blank piece of paper secured to the floor alongside the affected foot. If the child was unable to touch the wall with their heel on the ground, the paper was placed between the wall and the most anterior point of the knee. This resulted in a negative value. The angle measurements of the posterior leg remained the same. The measure marked on the blank piece of paper was placed in a sealed envelope until the end of the study. All distance measures were measured at the same time point at the completion of the study.

To measure the angle, the short arm of the digital inclinometer was placed flat against the posterior heel along the marked position. This was held in position, with the screen facing away from the rater for blinding until the rater stated they were pleased with the position. An 
217 independent research assistant noted the angle. The same protocol was performed with the 218 iPhone.

219

220 Between each measure, the child was allowed to rest as needed to relieve any discomfort

221 potentially caused by a sustained end range position and due to the child's attention span.

222

223 Data Analysis

224 All data analysis was conducted using SPSS Statistics 21 software package was used (IBM

225 Statistics, United States). Participants data were described in means (SD) and frequencies (\%).

226 The intra-rater reliability for each tool was determined using the intraclass correlation

227 coefficients (ICC) (Model 3,1) (two-way mixed with absolute agreement), the minimal

228 detectable change and standard error of the mean (SEM). The interrater reliability was

229 determined using ICCs (Model 3,1) (two-way mixed with absolute agreement), SEM and the

230 minimal detectable change. In consideration that joint stiffness may be present and impact the

231 first measure of testing session, an apriori decision was made that the second measure of each of

232 the raters were to be used for each of the tools. The concurrent validity of the parent/carer

233 population was explored using ICCs (Model 2,1) (Two-way random with absolute agreement).

234

235 The minimal detectable change is the minimal amount of change that is likely not to be due to

236 error. The SEM was used to calculate the minimal detectable change using the equation $1.96 \mathrm{x}$

237 SEM x $\sqrt{2} .{ }^{14}$ A smaller minimal detectable change is ideal as it improves confidence in

238 difference observed, however, it does not ensure clinical relevance. ${ }^{17}$

239

240 Based on an expected minimum ICC of 0.75 and a desired confidence interval (CI) width of 0.5

241 (i.e., the $95 \%$ CI of 0.50 to 1.00) for the intra-rater reliability analysis, it was estimated that the

242 minimum sample size should be 13 feet.

243

244 For the reliability or validity, an ICC value of $\geq 0.75$ with confidence interval of width 0.5 (range

$2450.5-1.0)$ was ideal. Ranges were determined, as per Portney and Watkins ${ }^{16}$ to report ICC data:

$246<0.5=$ poor reliability, 0.5 to $0.75=$ moderate reliability, 0.76 to $0.9=$ good reliability, and $>$

$2470.90=$ excellent reliability. 
248

249 All data was graphically represented on a Bland-Altmann plot. These plots provide a visual

250 spread, illustrative of differences between methods against the mean and assists with the decision

251 of whether the observed error is acceptable. ${ }^{16}$ It was used to assess the degree of agreement

252 between the two tools in all positions, by both raters, across the two timepoints.

253

254 Results

255 Participant characteristics

256 Twelve participants and their parents/carers met eligibility criteria with both parent and child

257 consenting to being involved in the study. Participants characteristics were recorded (Table 1).

258 Additionally, the carer filled out a purpose-built questionnaire (Additional file 8) to determine

259 the child's CTEV experience. Seven out of the twelve participants (58.3\%) had bilateral CTEV.

260 A slight gender bias existed with $66.7 \%$ being males (8:4), in keeping with expected gender

261 prevalence of CTEV.

262

263 TABLE 1 HERE

264

265 Study findings

266 Measures were taken on thirteen feet (Table 2). A negative recording on the knee to wall

267 measure (i.e. unable to touch the wall) was recorded for five (42.7\%) measures. Two hundred

268 and eight measures were recorded during the study.

269

270 The concurrent validity between the iPhone and digital inclinometer on flat and angled surface

271 (15 degrees) was determined prior to the study. The validity was excellent, indicated by an ICC

272 of 0.99 (95\% confidence interval -0.58 to 1.58$)$.

273

274 The intra-rater reliability between measures for the distance measure was excellent (ICC $=0.96-$ 275 0.99), very good for the digital inclinometer (ICC $=0.85-0.90)$ and good for the iPhone 276 measure app $(\mathrm{ICC}=0.75-0.90)$ (Table 3$)$. Inter-rater reliability between the clinician and 277 clinician in training was excellent using the distance measure (ICC $=0.95$ ), good when using the 278 inclinometer $(\mathrm{ICC}=0.80)$ and moderate for the iPhone measure application $(\mathrm{ICC}=0.68)($ Table 3$)$. 
280 The standard error of measurement (SEM) and minimal detectable change was determined for 281 the intra-reliability of each of the measures (Table 3). The minimal detectable change ranged 282 from 1.90 - 5.70 with the clinician in training's measures, using the digital inclinometer, having 283 the lowest minimal detectable change.

284

285 Concurrent validity between the clinician and parent/carer was good (ICC $=0.90$ ) for distance as 286 displayed by the Bland-Altmann plot below. The iPhone tool provided moderate validity 287 between the clinician and parent/carer $(\mathrm{ICC}=0.62)$.

288

289 TABLE 2 AND 3 HERE 
290 The Bland-Altmann plot (Figure 3) shows the agreement between the clinician and parents/carers 291 distance. All data points, except for one outlier, were between the limits of agreement. This 292 demonstrates the consistency and therefore concurrent validity of the measures.

293

294

295 FIGURE 3 HERE 


\section{Discussion}

297 This study is the first to explore the reliability of the WBLT within a CTEV population. The

298 WBLT is used by clinicians to assess ankle joint ROM and has been deemed reliable within 299 pathological paediatric populations, such as Charcot-Marie Tooth ${ }^{11}$, calcaneal apophysitis ${ }^{18}$ and 300 idiopathic toe walking. ${ }^{19}$ The current study followed the protocol of these previous studies,

301 which is an adapted version of the original WBLT by Bennell and Talbot. ${ }^{9}$ This study has

302 determined that identifying a change in ankle joint ROM using distance of toes from wall, and 303 inclinometer has good to excellent intra and inter-rater reliability and iPhone measure has good 304 intra-reliability. The measures can be used with credence by parents/carers to identify change in 305 ankle ROM, potentially indicating early CTEV relapse. As stated earlier, a reduction in ankle 306 joint ROM is one of the primary signs of relapse and early detection of change leads to earlier 307 intervention. 5,8

309 The literature reports the relapse involved with CTEV continues to be high. Children with CTEV 310 are reviewed by health professionals less frequently as they grow older; at a time when their risk

311 for relapse continues. ${ }^{5}$ Having parents/carers able to identify early changes in ankle joint ROM 312 improves monitoring abilities, detecting joint changes and relapses sooner for better outcomes.

313 Our sample was a population presenting to a metropolitan hospital were but given the ease of this 314 measure, it can be used anywhere. This is particularly significant in the Aboriginal and Torres 315 Strait Islander community where there is a much higher prevalence of CTEV. Given $11.9 \%$ of 316 Aboriginal and Torres Strait Islander people live in areas classified as very remote, and due to 317 inherent difficulties in receiving adequate health-care in remote areas, a heavier reliance on self318 monitoring is required. ${ }^{20}$ The use of simple tools like the distance or measure application can 319 allow people to identify concerns with their own health and seek more timely and appropriate 320 intervention.

321

322 The distance measure proved to be most reliable from the WBLT measure options reviewed, 323 potentially due to ease of application. However, this study determined the WBLT within a CTEV 324 population can be measured by a variety of people, in a variety of ways, with confidence. It is 325 noted the low minimal detectable change results across all measures suggest a small change in 326 measure cannot be attributed to an error in measurement and further boosts confidence that 
327 measurers are observing true change. These results are in keeping with previous investigations of

328 the reliability and validity WBLT in adult, paediatric and pathological populations. ${ }^{14}$

329

330 These outcomes should be considered against a number of limitations. Firstly, due to the CTEV

331 presentation, the children measured had feet with a soft heel and rounded lateral border (Figure

332 4). This potentially increased the difficulty of obtaining consistent measures.

333

334

335

336

337

338

339

340

341

342

343

344

345

346

347

348

349

350

351

352

353

354

355

356

357

\section{FIGURE 4 HERE}

The inquisitive nature of the children along with the repetitive nature of three measuring tools, lead to frequent movement, with children attempting to change body position to gain a better view of what was occurring. This occasionally meant there was some movement of the foot, requiring realignment. It is also important to mention also that this study only measured ankle dorsiflexion. A relapse of CTEV could, potentially, occur in multiple planes due to the nature of the condition. It is important that this is deliberated when considered for application. This study only measured the reliability of an iPhone with regards to phone type. The results are therefore most relevant to Apple users. Although the distance measure can be used by all and is most reliable, there is potential to assess this measure using different technologies. Future studies are required for the long term follow up of the use of the WBLT by carers as a self-monitoring tool. This should be followed in relation to reported relapse identification. Particularly in remote areas to determine the efficiency of the tool.

Converse to much of the literature the clinician in training and parent/carer were observed to have moderately smaller SEMs to the clinician. ${ }^{22}$ This may reflect care and concentration of the novice users and suggests future studies should consider using more than one representative for each user group involved.

Future studies should involve the development and testing of a WBL protocol for use at home by parents/carers in relation to the sensitivity and specificity of the measure. This protocol could 
358 involve a prospective long-term investigation prior to determining if the WBLT measure alone is 359 competent in detecting a CTEV relapse in the home setting.

360

361 Conclusion

362

363 The WBLT within a paediatric CTEV population has good to excellent reliability when used by

364 either a clinician, clinician in training or parent/carer, for distance from the wall, or the angle of

365 the posterior lower leg when using an inclinometer or iPhone (intra-reliability only). Good

366 concurrent validity is also demonstrated for the distance measure. The results of this study are

367 encouraging as a tool for increasing self-monitoring of this condition and potential earlier

368 detection of relapses. This will be particularly useful in remote areas with limited health-care

369 services. Ankle dorsiflexion is, however, just one of the signs of relapse and it would be prudent

370 for clinicians to consider other signs and symptoms prior to diagnosis. Future studies should aim

371 to develop a protocol for this measure at home with parents and test the effectiveness of relapse

372 prediction and associated outcomes.

373 
374 Acknowledgements

375 Thank you to the University of South Australia and their library staff for their assistance with

376 the search of literature. Thank you also to the Women's and Children's Hospital in Adelaide for

377 the use of their resources.

378

379

380

381

382

383

384

385

386

387

388

389

390

391

392

393

394

395

396

397

398

399

400

401

402

403

404

405

406

407

408

409

410

411

\section{References}

1. Ansar A, Rahman AE, Romero L, Haider MR, Rahman MM, Moinuddin M, Siddique MAB, Mamun MA, Mazumder T, Pirani SP, Mathias RG, Arifeen SE, Hoque DME . Systematic review and meta-analysis of global birth prevalence of clubfoot: a study protocol. BMJ open. 2018;8(3):e019246.

2. Symeonidis P, Christodoulou A, Mareti E, Eleftherochorinou A, Gkourani S, Mbeslikas T, Kyrkos, J. Patient-Based Long-Term Results of the Surgical Treatment of Congenital Talipes Equinovarus. Foot \& ankle specialist. 2016;9(3):258-64.

3. Smythe T, Kuper H, Macleod D, Foster A, Lavy C. Birth prevalence of congenital talipes equinovarus in low-and middle-income countries: a systematic review and meta-analysis. Tropical medicine \& international health. 2017;22(3):269-85.

4. Anand A, Sala DA. Clubfoot: Etiology and treatment. Indian journal of orthopaedics. 2008;42(1):22.

5. Ponseti IS, L Morcuende, JA Pirani, S Mosca, V Penny, N Dietz, F Herzenberg, JE Weinstein, S Steenbeek, M Clubfoot: Ponseti Management Third Edition. 2009.

6. Bergerault F, Fournier J, Bonnard C. Idiopathic congenital clubfoot: Initial treatment. Orthopaedic Traumatol Surgical Research. 2013;99(1 Suppl):S150-9.

7. Nogueira MP, Farcetta M, Fox MH, Miller KK, Pereira TS, Morcuende JA. Treatment of congenital clubfoot with the Ponseti method: the parents' perspective. Journal Pediatric Orthopaedics B. 2013;22(6):583-8.

8. Sangiorgio SN, Ebramzadeh E, Morgan RD, Zionts LE. The Timing and Relevance of Relapsed Deformity in Patients with Idiopathic Clubfoot. Journal AM Academy Orthopaedic Surgery. 2017;25(7):536-45.

9. Bennell K, Talbot R, Wajswelner H, Techovanich W, Kelly D, Hall A. Intra-rater and interrater reliability of a weight-bearing lunge measure of ankle dorsiflexion. Australian Journal of physiotherapy. 1998;44(3):175-80.

10. Bennell K, Khan KM, Matthews B, De Gruyter M, Cook E, Holzer K, Wark JD. Hip and ankle range of motion and hip muscle strength in young female ballet dancers and controls. British journal of sports medicine. 1999;33(5):340-6.

11. Rose KJ, Burns J, North KN. Factors associated with foot and ankle strength in healthy preschool-age children and age-matched cases of Charcot-Marie-Tooth disease type 1A. Journal of child neurology. 2010;25(4):463-8. 
412 12. Lozano P, Houtrow A. Supporting self-management in children and adolescents with 413 complex chronic conditions. Pediatrics. 2018;141(Supplement 3):S233-S41.

414 13. Williams CM, Caserta AJ, Haines TP. The TiltMeter app is a novel and accurate 415 measurement tool for the weight bearing lunge test. Journal of Science and Medicine in Sport. 416 2013;16(5):392-5.

417 14. Banwell HA, Uden H, Marshall N, Altmann C, Williams CM. The iPhone Measure app level 418 function as a measuring device for the weight bearing lunge test in adults: a reliability study.

419 Journal of Foot and Ankle Research. 2019;12(1):37.

420 15. Franko OI, Tirrell TF. Smartphone app use among medical providers in ACGME training 421 programs. Journal of medical systems. 2012;36(5):3135-9.

422 16. Portney LG, Watkins MP. Foundations of Clinical Research: Applications to Practice. Third 423 edition ed. Connecticut: Appleton \& Lange; 2015.

424 17.Turner, D Schunemann HJ, Griffith, LE Beaton DE Griffiths, AM Critch, JN Guyatt, GH. The 425 minimal detectable change cannot reliably replace the minimal important difference. Journal of 426 Clinical Epidemiology. 2010; 63(1):28-36

427 18. James AM, Williams CM, Luscombe M, Hunter R, Haines TP. Factors associated with pain 428 severity in children with calcaneal apophysitis (sever disease). The Journal of pediatrics. 429 2015;167(2):455-9.

430 19. Williams C, Tinley PD, Curtin M, Nielsen S. Foot and ankle characteristics of children with 431 an idiopathic toe-walking gait. Journal of the American Podiatric Medical Association.

432 2013;103(5):374-9.

433 20. Australian Bureau of Statistics. Estimates of Aboriginal and Torres Strait Islander 434 Australians. In: Statistics ABo, editor. Canberra2018.

435 21. White KK, Bouchard M, Goldberg MJ. Common Neonatal Orthopedic Conditions. Avery's 436 Diseases of the Newborn: Elsevier; 2018. P. 1438-49. e3.

437 22. Diamond, JE Mueller, MJ Delitto, A Sinacore, DR. Reliability of Diabetic Foot Evaluation; 438 Physical Therapy; 1989; 69: 797-802

439

440

441

442

443

444

445

446

447

448

449

450

451

452 
453

454

455

456

457

458

459

460

461

462 
Table $\mathbf{1}$ (on next page)

Table 1 Participant Data 
Table 1: Participant data

\begin{tabular}{lll} 
Characteristic & Mean $( \pm$ SD) & Range \\
\hline Age $($ years $)$ & $7.00(+/-1.80)$ & $5-10$ \\
Weight $(\mathrm{kg})$ & $22.90(+/-7.60)$ & $15-39$ \\
Height $(\mathrm{cm})$ & $121.90(+/-14.60)$ & $102-148$ \\
Shin length $(\mathrm{cm})$ & $28.20(+/-4.90)$ & $21-35$ \\
Foot length $(\mathrm{cm})$ & $16.60(+/-2.80)$ & $14-22$ \\
Pirani score (from birth) & $5.00(+/-1.03)$ & $3-6$
\end{tabular}


Table 2 (on next page)

Raw Measurements

Table two shows measure two taken by each rater for each participant. 
Table 3: Table of raw measurements

TABLE OF RAW RESULTS

\begin{tabular}{|c|c|c|c|c|c|c|c|c|c|c|}
\hline \multirow[b]{2}{*}{ Foot \# } & \multicolumn{5}{|c|}{ Distance score (mm) } & \multicolumn{2}{|c|}{$\begin{array}{c}\text { Angle at back of tibia } \\
\text { (inclinometer) (degrees) }\end{array}$} & \multicolumn{3}{|c|}{$\begin{array}{c}\text { Angle at back of tibia (iPhone compass } \\
\text { app) (degrees) }\end{array}$} \\
\hline & & Clinician & & $\begin{array}{l}\text { Clinician in } \\
\text { training }\end{array}$ & Parent/Carer & Clinician & $\begin{array}{l}\text { Clinician in } \\
\text { training }\end{array}$ & Clinician & $\begin{array}{l}\text { Clinician in } \\
\text { training }\end{array}$ & Parent/Carer \\
\hline & 1 & & -40 & -71 & -22 & 19.4 & 19.4 & 16 & 16 & 17 \\
\hline & 2 & & -30 & -29 & -28 & 29.6 & 28.8 & 11 & 25 & 25 \\
\hline & 3 & & -10 & 0 & 5 & 26.2 & 22.8 & 23 & 21 & 24 \\
\hline & 4 & & 10 & 0 & 0 & 27.5 & 27.5 & 27 & 28 & 28 \\
\hline & 5 & & 19 & 12 & 21 & 23.9 & 20.7 & 24 & 20 & 24 \\
\hline & 6 & & -11 & -18 & -3 & 23.8 & 22.4 & 26 & 22 & 23 \\
\hline & 7 & & 6 & 0 & 6 & 19.8 & 24 & 25 & 25 & 20 \\
\hline & 8 & & 0 & 0 & 15 & 26 & 23 & 27 & 24 & 28 \\
\hline & 9 & & -1 & -16 & -12 & 20.6 & 19.1 & 17 & 18 & 18 \\
\hline & 10 & & 32 & 40 & 66 & 27.1 & 28.2 & 26 & 28 & 33 \\
\hline & 11 & & 17 & 23 & 35 & 17.1 & 24.3 & 16 & 19 & 24 \\
\hline & 12 & & 29 & 27 & 50 & 27.2 & 27.8 & 25 & 26 & 29 \\
\hline & 13 & & 16 & 22 & 19 & 25.6 & 26 & 24 & 26 & 24 \\
\hline
\end{tabular}


Table 3 (on next page)

Study Results

Table 3 shows statistical results from raw data collected 
Table 3: Study results

INTRA-RATER RELIABILITIES

\begin{tabular}{|c|c|c|c|c|c|c|}
\hline & Rater & Mean (SD) & ICC & $95 \% \mathrm{CI}$ & SEM & MDC \\
\hline \multirow{2}{*}{$\begin{array}{l}\text { Digital inclinometer } \\
\text { (degrees) }\end{array}$} & Clinician & $-1.50(+/-2.30)$ & 0.87 & $0.52,0.96$ & 0.83 & 2.30 \\
\hline & Clinician in training & $0.90(2.20)$ & 0.90 & $0.68,0.97$ & 0.70 & 1.93 \\
\hline \multirow[t]{3}{*}{ iPhone (degrees) } & Clinician & $-0.50(4.10)$ & 0.75 & $0.16,0.92$ & 2.05 & 5.68 \\
\hline & Clinician in training & $0.30(2.60)$ & 0.90 & $0.68,0.97$ & 0.82 & 2.28 \\
\hline & $\mathrm{PC}$ & $-1.80(2.40)$ & 0.90 & $0.49,0.97$ & 0.76 & 2.10 \\
\hline \multirow[t]{3}{*}{ Distance (mm) } & Clinician & $-2.20(10.00)$ & 0.96 & $0.86,0.99$ & 2.00 & 5.54 \\
\hline & Clinician in training & $-2.00(7.10)$ & 0.98 & $0.96,0.99$ & 1.00 & 2.78 \\
\hline & $\mathrm{PC}$ & $0.43(7.80)$ & 0.97 & $0.88,0.99$ & 1.35 & 3.74 \\
\hline \multicolumn{7}{|c|}{ INTER-RATER RELIABILITIES } \\
\hline & Raters & Mean (SD) & ICC & $95 \% \mathrm{CI}$ & & \\
\hline $\begin{array}{l}\text { Digital inclinometer } \\
\text { (degrees) }\end{array}$ & $\begin{array}{l}\text { Clinician/clinician } \\
\text { in training }\end{array}$ & $-0.01(2.90)$ & 0.80 & $0.32-0.94$ & & \\
\hline iPhone (degrees) & $\begin{array}{l}\text { Clinician/clinician } \\
\text { in training }\end{array}$ & $-0.90(4.60)$ & 0.68 & $0.06-0.90$ & & \\
\hline Distance (mm) & $\begin{array}{l}\text { Clinician/clinician } \\
\text { in training }\end{array}$ & $3.60(11.10)$ & 0.95 & $0.84-0.98$ & & \\
\hline \multicolumn{7}{|c|}{ CRITERION VALIDITY } \\
\hline & Raters & Mean (SD) & ICC & $95 \% \mathrm{CI}$ & & \\
\hline iPhone (degrees) & Clinician/PC & $-2.3(4.90)$ & 0.62 & $-0.11,0.88$ & & \\
\hline Distance (mm) & Clinician/PC & $-8.8(12.80)$ & 0.89 & $0.58,0.97$ & & \\
\hline
\end{tabular}

Abbreviations: SD - standard deviation; ICC - intraclass correlation coefficient; CI - confidence interval; SEM - standard error of measurement; MDC minimal detectable change; $\mathrm{PC}-$ parent/carer 
Figure 1

Figure 1 Position of weight bearing lunge test with iPhone positioning and screen positioning demonstrated (authors own image) 


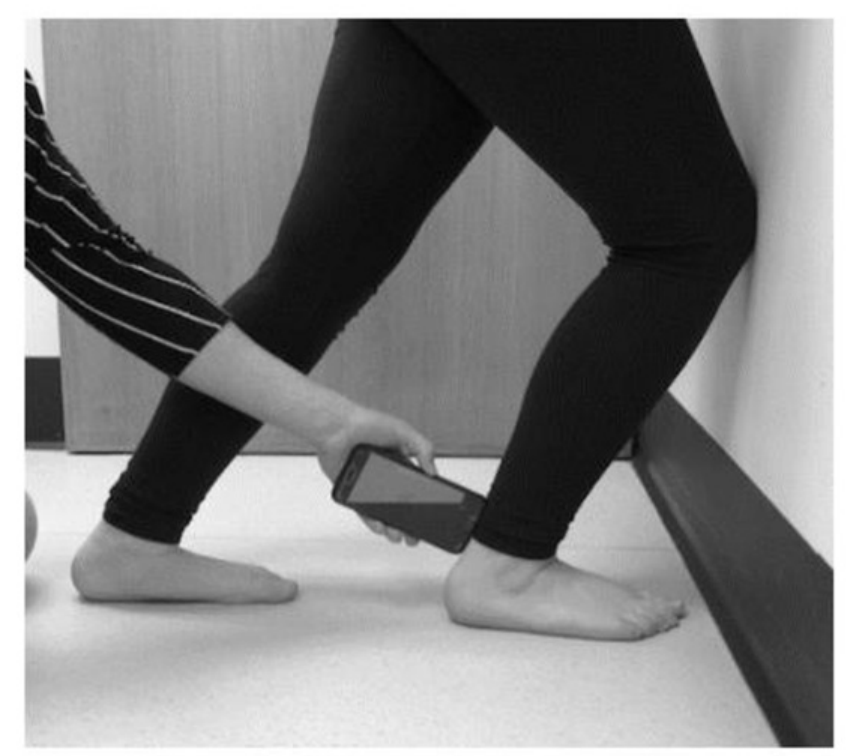


Figure 2

Figure 2 Process of weight bearing lunge test 
1. Participants were asked to place their affected/chosen foot in front of wall with toes pointing to wall

- Knee was to be aligned over the second toe and aimed at a line on the wall.

-The participants were asked to place both hands on the wall in front of them.

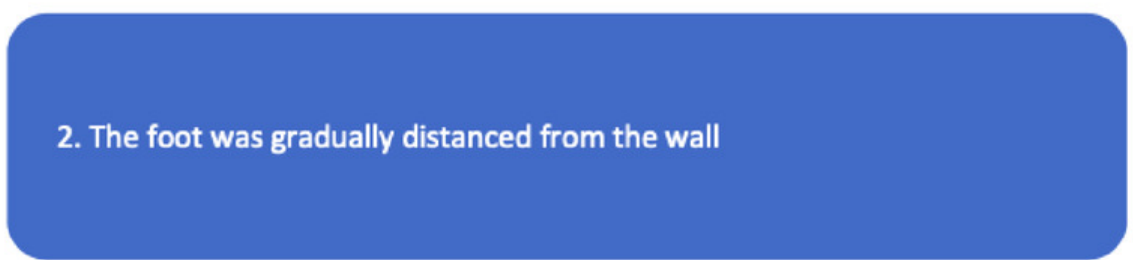

-The foot was moved to obtain the furthest possible distance from the wall with knee contacting wall if possible*

-This was done with the heel remaining in contact with the ground.

3. At full lunge position, with the heel remaining in contact with the ground and knee contacting wall if possible*, each rater recorded a single measure of range of motion with relevant tool and then repeated procedure on second occasion. 
Figure 3

Figure 3 Bland-Altmann demonstrating agreement between clinicians and parents/carers distance measure (concurrent validity) 


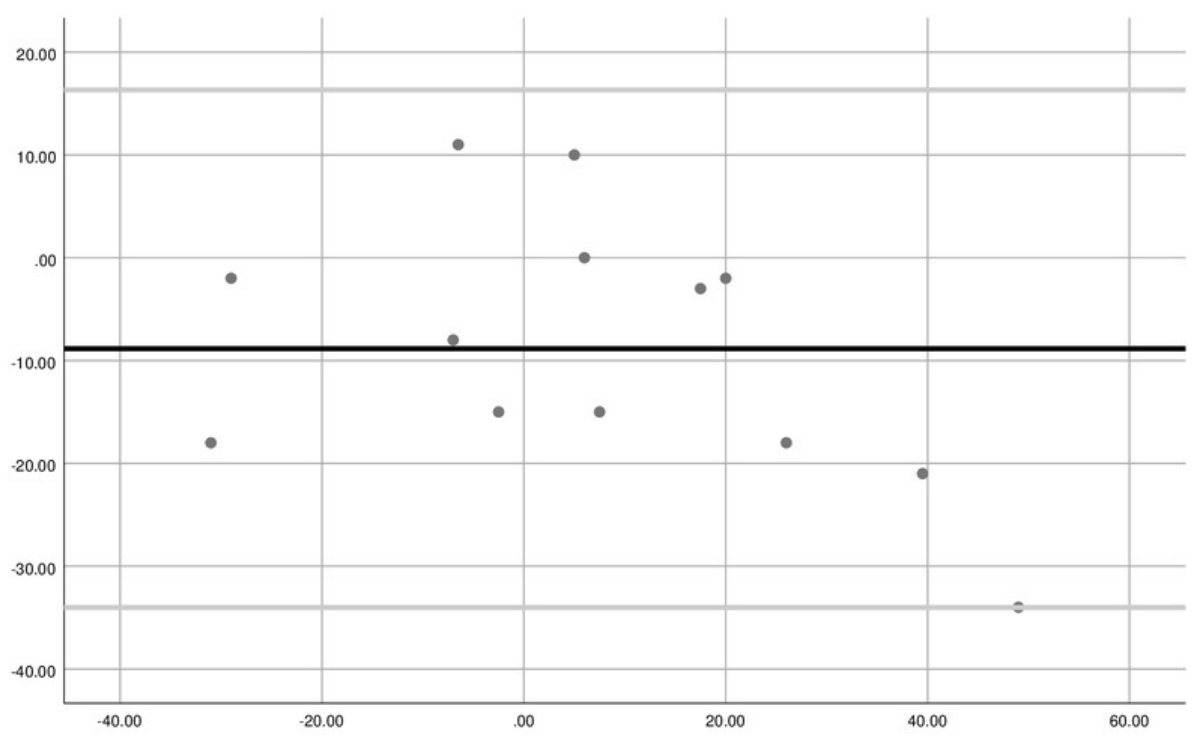

Page 1 
Figure 4

Figure 4 Example of clubfoot with rounded lateral border 


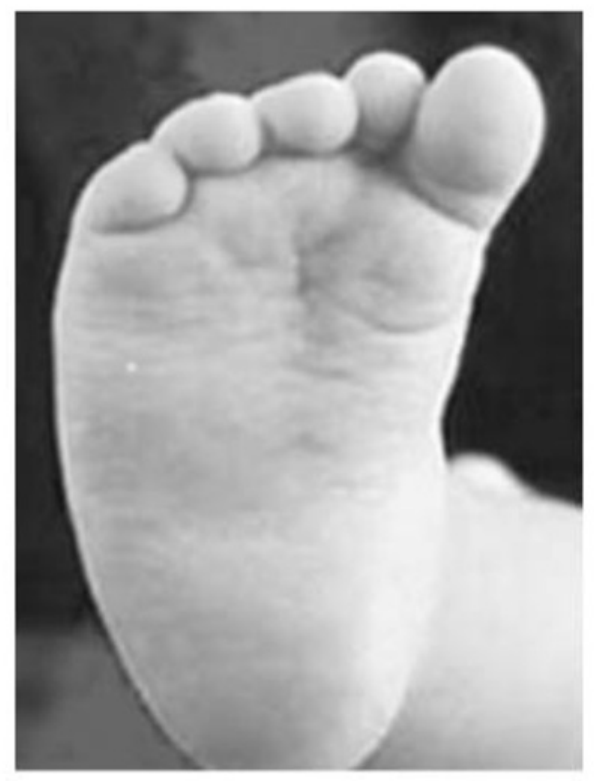

